\title{
Révision tarifaire: TARCO (TARMED CONSENSUS) est sur les rails
}

\section{Urs Stoffel}

Dr méd., membre du Comité central de la FMH, responsable du département Médecine et tarifs ambulatoires

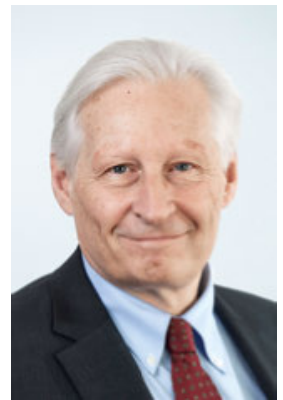

TARCO est la seule issue au tarif imposé dès le $1^{\mathrm{er}}$ janvier 2018 par l'Etat. En cherchant l'expression «être sur les rails» sur Google, on trouve différentes interprétations comme "trouver une piste sérieuse» ou "être dans la bonne direction». Le projet TARCO est effectivement sur la bonne voie et proche du but!

Institué par l'Assemblée des délégués de la FMH en tant qu'organe de décision suprême du projet TARCO, l'organe de pilotage, le "COCKPIT", se compose de représentants de toutes les organisations faîtières et organisations de base de la FMH. Lors de sa séance, le 29 novembre 2017, le COCKPIT a approuvé plus de $90 \%$ de la nomenclature de la structure tarifaire. Reste désormais à éliminer les dernières divergences ces prochains jours et à finaliser les derniers chapitres interdisciplinaires.

La prochaine étape majeure avant le dépôt conjoint de la structure tarifaire TARCO consiste à négocier avec les partenaires tarifaires; dans certains domaines, les premières négociations ont déjà débuté ou sont en passe de l'être.

Depuis le feu vert de l'Assemblée des délégués de la FMH au projet TARCO en janvier 2017, plus de 150 délégués tarifaires et experts, regroupés dans plus de

\section{TARCO est la seule issue au tarif imposé dès le $1^{\text {er }}$ janvier 2018 par l'Etat.}

40 groupes de travail, sont à l'œuvre. TARCO se fonde sur la version ats-tms 1.0 (TARVISION) du tarif, rejetée en juin 2016 par les membres de la FMH en votation générale. L'ensemble des chapitres ont été retravaillés en concertation avec toutes les sociétés de discipline médicale et harmonisés conformément aux principes tarifaires de base du projet TARCO.

Chaque chapitre a ensuite été mis à la disposition de toutes les sociétés lors de l'une des quatre phases de consultation afin qu'elles puissent se prononcer. Enfin, les retours ont été analysés et intégrés au tarif lorsque cela était indiqué.

Par rapport au TARMED encore en vigueur (version 1.08_BR) avec ses 4519 positions, le tarif ambulatoire TARCO ne compte plus que quelque 2700 positions, soit $40 \%$ en moins. "Oui, mais c'est encore beaucoup trop, il faut simplifier", diront certains. Rappelons cependant que le TARMED est un tarif à la prestation devant

\section{Dans certains domaines, les premières négociations avec les partenaires tarifaires ont déjà débuté.}

refléter toutes les prestations ambulatoires obligatoires selon l'ordonnance sur les prestations de l'assurance des soins (OPAS). Si, pour certains, la structure tarifaire TARMED est beaucoup trop détaillée et complexe, pour d'autres, elle manque de transparence et on ne sait plus ce qui a été fait. Deux exigences - transparence d'une part, simplification de l'autre - diamétralement opposées. Ce que nous savons en revanche, c'est que 97\% du volume des coûts générés aujourd'hui par le TARMED proviennent de seulement 200 positions.

L'appel à davantage de forfaits dans le tarif ambulatoire TARMED a été très bien saisi par la FMH, qui est prête à intégrer des forfaits là où cela est possible et indiqué. Pour cela, certaines prestations isolées devraient être regroupées à des fins de simplification. La base de tout forfait est cependant un tarif à la prestation approprié et conforme aux règles de l'économie d'entreprise. Ce n'est qu'avec une bonne connaissance des prestations dont on parle que l'on peut ensuite les regrouper de manière transparente dans des forfaits. Nous avons bon espoir de pouvoir mettre sur pied un tarif orienté vers l'avenir, susceptible de réunir un consensus et avant tout approprié. Nous sommes en bonne voie! 